\title{
Morfotectónica de la falla Abriaquí y sismicidad histórica asociada con el sismo de 1903 en Frontino, Antioquia
}

\author{
Santiago Noriega-Londoño,*, José Humberto Caballero-Acosta² \\ ${ }^{1}$ Departamento de Materiales y minerales, Facultad de Minas, Universidad Nacional de Colombia, Medellin, Colombia \\ ${ }^{2}$ Departamento de Geociencias y medio ambiente, Facultad de Minas, Universidad Nacional de Colombia, Medellin, Colombia
}

\begin{abstract}
Resumen
En el sistema orogénico de los Andes del Norte se han desarrollado importantes fallas activas que revelan un grado moderado a alto de amenaza sísmica en el borde noroccidental de Colombia. Este estudio presenta un análisis morfotectónico que señala la ocurrencia de deformaciones recientes a lo largo de la falla Abriaquí e introduce nuevos datos de sismicidad histórica que permiten la atribución preliminar del sismo de 1903 en Frontino a esta estructura, y ubica la edad de su último desplazamiento en periodos históricos.
\end{abstract}

Palabras clave: Frontino, sismicidad histórica de Colombia, falla Abriaquí, morfotectónica, neotectónica, Antioquia.

Morphotectonics of the Abriaquí fault, historical seismicity and their relation with the 1903 earthquake in Frontino, Antioquia

\begin{abstract}
Major active faults have developed in the North Andean orogenic system that reveal moderate to high seismic hazard in the northwestern border of Colombia. This study presents a morphotectonic analysis of recent deformations along the Abriaquí fault and introduces new historical seismicity data that allows for preliminarily assigning the 1903 earthquake in Frontino to this structure, and modifying the age of its last displacement to historical time periods.
\end{abstract}

Key words: Frontino, historical seismicity of Colombia, Abriaquí fault, morphotectonics, neotectonics, Antioquia.

\section{Introducción}

Este articulo presenta los resultados parciales del proyecto "Evidencias preliminares de tectónica activa entre la cuenca media del Río El Cerro y la vereda El Chuscal, Frontino Antioquia, Cordillera Occidental”, desarrollado por medio del programa de Jóvenes Investigadores de Colciencias - 2012. Se exponen, específicamente, nuevos datos tanto de sismicidad histórica como de morfotectónica, los cuales permiten reevaluar las condiciones neotectónicas de la falla Abriaquí.

El objetivo del estudio consistió en revisar y actualizar la base de datos de sismicidad histórica en el occidente colombiano, en particular lo relativo al evento de 1903, y contrastarla con los resultados del análisis morfotectónico de la falla Abriaquí, la cual no ha sido considerada hasta ahora como una estructura potencialmente sismogénica. Se presentan nuevos datos neotectónicos a escala local que permitan refinar el catálogo de fallas y pliegues cuaternarios de Colombia y actualizar las bases de datos de evaluación de amenaza sísmica en el occidente colombiano.

La falla Abriaquí es una estructura transpresiva de aproximadamente $34 \mathrm{~km}$ de longitud, de componente sinistral/ inversa, que se extiende al suroccidente del municipio de Frontino con tendencia principal en sentido $\mathrm{N}^{\circ} 0^{\circ} \mathrm{O}$ que varía a NNO en el tramo norte. Esta falla posee una expresión geomorfológica bien definida, representada por silletas, escarpes y corrientes deflectadas. Page (1986) reporta deformaciones en depósitos cuaternarios que reposan sobre esta estructura, y le asigna un grado de actividad moderado con tasas de desplazamiento entre 0,2 y 1,0 mm/año y edades por debajo de 1,6 Ma para el último movimiento. Además, en el área de influencia de esta estructura se han reportado sismos históricos importantes, como el de 1903, el cual generó daños graves en el municipio de Frontino, aunque en los municipios vecinos los efectos fueran considerablemente menores, lo que indica, quizá, el carácter local y superficial del evento.

Los resultados obtenidos sugieren que la falla Abriaquí ha tenido desplazamientos más recientes, ocurridos al menos en el Holoceno, y que probablemente el sismo de 1903 se produjo sobre esta estructura, lo cual plantea su relevancia en términos sismogénicos; sin embargo, se hace necesario diseñar y ejecutar campañas de exploración paleosismológica que permitan estimar con un mayor grado de detalle la historia sísmica de esta falla.

\footnotetext{
*Correspondencia:

Santiago Noriega Londoño; santiageo@gmail.com

Recibido: 6 de agosto de 2014

Aceptado: 16 de febrero de 2015
} 


\section{Marco geotectónico}

El borde noroccidental de Colombia se sitúa en un complejo sistema orogénico de edad Meso-Cenozoico conformado por eventos sucesivos de acreción de bloques y terrenos exóticos involucrados en el régimen compresivo de las placas de Nazca-Farallones, Caribe y Suramérica, y de los bloques Panamá-Chocó (BPC) y Norandino (BNA).

El proceso de acreción y engrosamiento de la margen puede establecerse desde finales del Cretáceo, en principio por efecto de la migración de la placa Caribe en sentido NNE (Kennan \& Pindell, 2009; Cediel, et al., 2011); esta fase está representada por el plegamiento y la formación de fallas dextrales en sentido NS dentro del grupo Cañasgordas, como las fallas San Ruperto y Portachuelo. Un segundo evento importante corresponde a la partición de la placa Farallones hacia el Mioceno medio (Chicangana, 2005; López, et al., 2006), indicado una variación del régimen tectónico con un vector de deformación en sentido OE, que coincide con el acercamiento y posterior acreción del BPC y la placa Nazca.

El evento geodinámico más relevante, generador del régimen tectónico dominante en el presente, corresponde a la colisión del bloque BPC con el bloque BNA hacia finales del Mioceno (Duque-Caro, 1990; Taboada, et al., 2000; Vargas, et al., 2002; Vargas \& Mann, 2013), lo que dio lugar al último pulso de la orogenia andina (fase Eu-andina, Van der Hammen, 1960). De esta forma, la indentación tectónica del BPC sobre el BNA constituye el evento geodinámico del cual se deriva el marco neotectónico del noroccidente colombiano.

\section{Marco geológico y tectónico}

La configuración geológica del occidente colombiano comprende una serie de unidades litotectónicas de afinidad oceánica que han sido acrecionadas a la margen oriental del BNA a lo largo del periodo Meso-Cenozoico (Restrepo \& Toussaint, 1988; Cediel, et al., 2011). Las rocas oceánicas se agrupan en dos grandes bloques (Figura 1). El primero corresponde a ofiolitas desmembradas e intensamente deformadas de edad cretácica que conforman el grupo Cañasgordas, mientras que el segundo corresponde a un arco de islas oceánico compuesto por el batolito de Mande y el complejo Santa Cecilia - La Equis, que constituyen el borde oriental del BPC. Estos bloques se encuentran en contacto por la falla de Dabeiba-Pueblo Rico (Figura 2), la cual se prolonga al norte y toma el nombre de falla Uramita, en tanto que en el extremo oriental se encuentra intruida localmente por pequeños cuerpos plutónicos correspondientes al cinturón magmático del Mioceno medio, el cual se extiende a lo largo del eje de la Cordillera Occidental. Por último, en el fondo de los valles se han concentrado espesos depósitos aluviales y aluviotorrenciales de edad cuaternaria, cubriendo parcialmente estructuras geológicas como las fallas Abriaquí, Murrí-Mutatá y San Ruperto, entre otras.

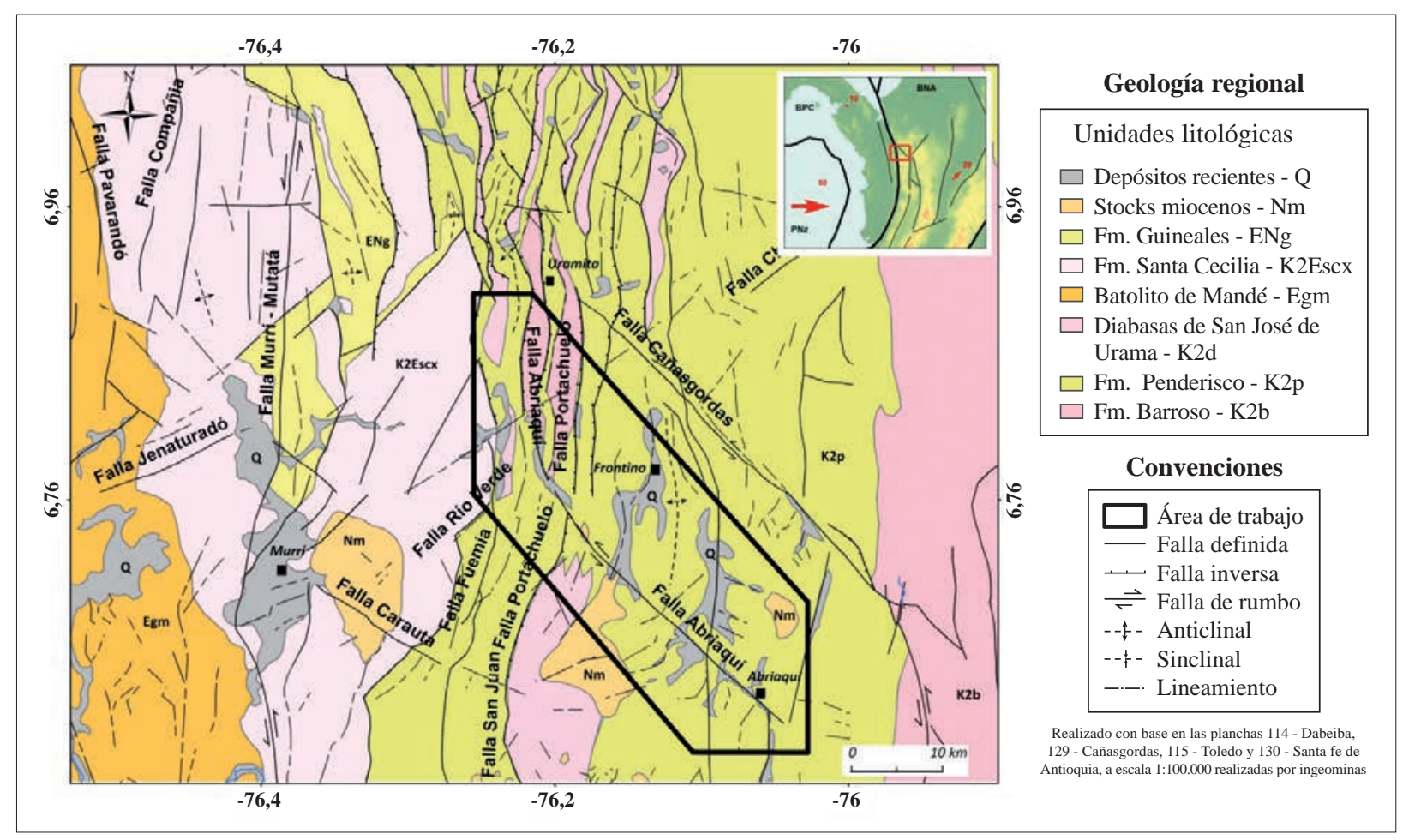

Figura 1. Geología regional y tectónica del área de estudio. Modificado de la cartografía geológica de Ingeominas a escala 1:100.000. Planchas 114 - Dabeiba y 129 - Cañasgordas 


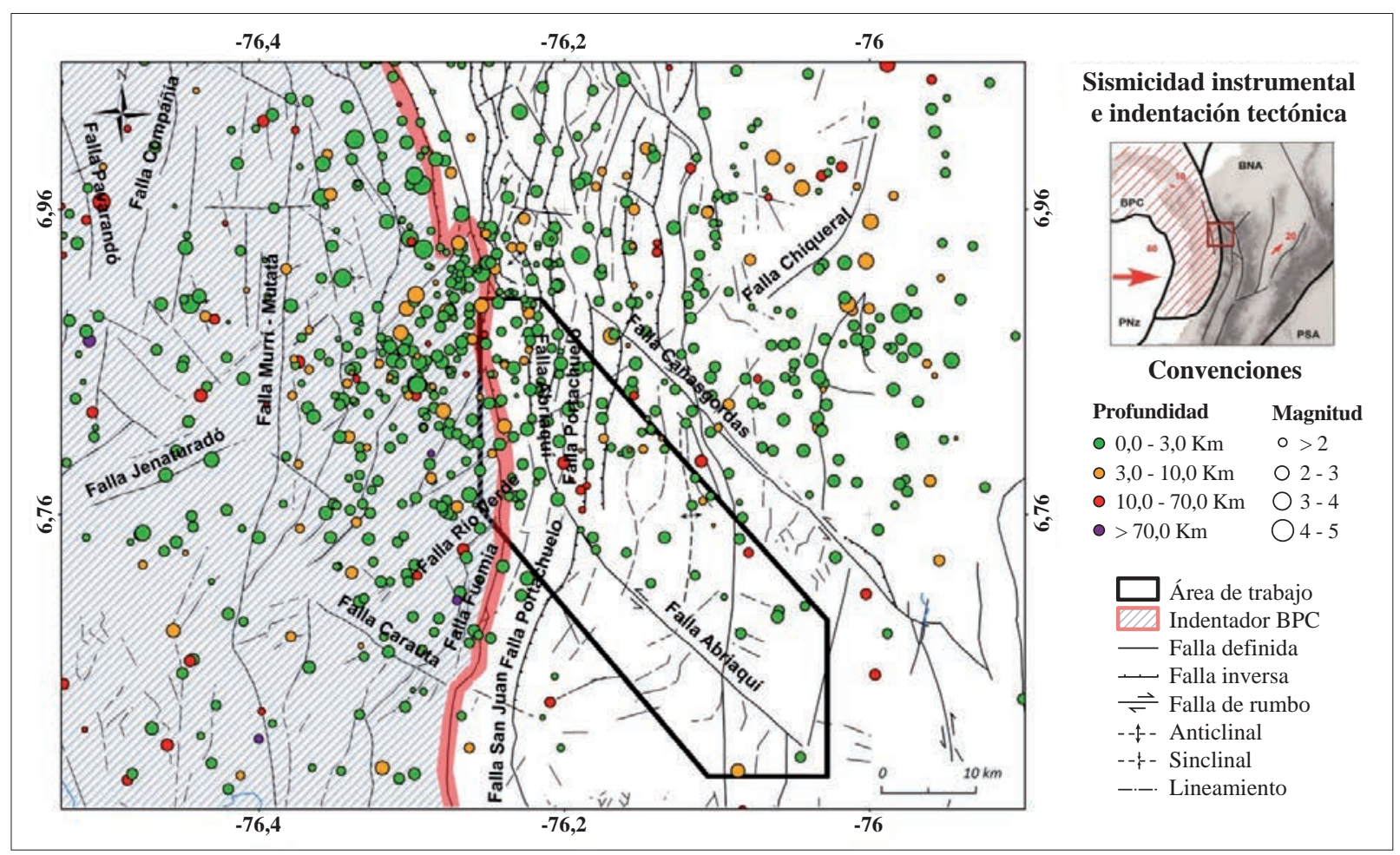

Figura 2. Contexto geodinámico del área de estudio, sismicidad instrumental y esquema de indentación tectónica

La deformación frágil existente en la zona, derivada de las fases finales de acreción, viene definida por lineamientos y sistemas de fallas sinistrales de orientación NO y componentes inversos, y por fallas inversas de orientación NS. Entre las primeras se incluyen las fallas Abriaquí y Cañasgordas, y fallas menores como las de Portachuelo y Morrogacho en la zona occidental y las de Arma y La Mansa en el extremo oriental. Las estructuras de orientación NS, por su parte, vienen representadas por los sistemas de fallas de CaucaRomeral, Herradura-San Ruperto, Portachuelo, DabeibaPueblo Rico, Murrí-Mutatá y Murindó, que generalmente exhiben un comportamiento predominantemente inverso con componente lateral izquierdo.

Las relaciones temporales de estos dos sistemas indican que las fallas de orientación NO constituyen las estructuras más recientes, puesto que desplazan y controlan la deformación de las fallas NS, como lo sugiere el desplazamiento de la falla Portachuelo por la falla Abriaquí, observado directamente en campo, y la deformación de fallas cretácicas en cercanías del sistema Cauca-Romeral en respuesta a la colisión del BPC (López, et al., 2006; Ramírez, et al., 2012), mientras que las fallas de orientación NS, sufren una reactivación e inversión como respuesta al evento de acreción tardío.

\section{Materiales y métodos}

El problema planteado se abordó mediante la aplicación de marcadores morfotectónicos y el análisis de registros de sismicidad histórica. Las características morfotectónicas y las áreas de deformación reciente o activa a escala local se obtuvieron mediante la aplicación de técnicas de fotointerpretación geológica en una escala de 1/10.000, cartografía geológica en escalas de 1/20.000 y 1/3.000, análisis de capas de altitud, pendiente y aspectos extraídas del STRM (Shuttle Radar Topography Mission), con resolución de $30 \mathrm{~m}$ a lo largo de la falla Abriaquí, y perfiles topográficos extraídos del modelo digital de elevaciones (MDE) mediante un sistema de información geográfica ArcGIS 10.0. A escala local, se realizaron perfiles longitudinales usando un GPS Garmin con error promedio de \pm 3 metros para cada punto medido. Al mismo tiempo, en el análisis de la sismicidad histórica se siguió la metodología clásica de Ingeominas, consistente en la revisión de archivos históricos que, en este caso, reposaban en la casa de la cultura y en el despacho parroquial del municipio de Frontino; la evaluación macrosísmica apoyada en los análisis de Cifuentes \& Sarabia (2009) para el evento de 1903, y la estimación de parámetros sismológicos como la localización epicentral, la magnitud y la profundidad, así como los datos de sismicidad instrumental suministrados por la Red Sismológica Nacional de Colombia (RSNC) para un periodo de 20 años entre 1993 y 2013. Esta información se analizó y luego se integró mediante el sistema de información geográfica para establecer relaciones espaciales entre las variables. Por último, se incluyeron relaciones entre modelos análogos de indentación tectónica y los datos disponibles en la literatura científica relacionada con la colisión del BPC. 


\section{Resultados}

\section{Sismicidad histórica}

$\mathrm{Al}$ enfrentar el estudio de la neotectónica desde la perspectiva de la sismicidad histórica es necesario partir de las dos premisas señaladas por Espinosa (2003): i) la sismicidad colombiana es principalmente preinstrumental, por lo tanto, la inclusión de los catálogos históricos se convierte en una herramienta indispensable, y ii) la identificación de fuentes sísmicas es efectiva mediante la superposición de epicentros y fallas activas.

Los registros de sismicidad histórica disponibles indican la ocurrencia de sismos sentidos en el noroccidente colombiano en los años de 1792, 1888, 1903, 1952, 1981, 1987 y 1992, (Ramírez, 1975; Arias \& Tejada, 1987; Caballero, 1991; Velásquez, et al., 1993), entre los cuales el evento de 1903 reporta los efectos más notables en el municipio de Frontino, con alteraciones en el relieve y daños directos sobre la población, por lo que el análisis de sismicidad histórica se concentra particularmente en dicho evento.

Para este evento en particular, Ramírez (1975) reporta que:

“... el movimiento fue al principio vertical y luego fuertemente horizontal en Frontino, donde fue el epicentro; las tejas cayeron de los techos y hubo muchas grietas en los muros. Volvió a repetir a las 9 am produciendo daños y pánico."

White (1959), por su parte, indica que:

"Se experimentaron dos de aquellos movimientos, los cuales podemos llamar locales, pues su epicentro estuvo dentro de la serie de cordilleras formadas por el Plateado de El Cerro, el Cerro de Muzinga [sic], de 3800 mtrs. de altura sobre el nivel del mar, Morro Pelado, el Cerro de La Horqueta, el Páramo de Frontino y los cerros de El Paramillo, Tres Morros y el León por el lado norte. Más allá de estos lugares, los sismas [sic] fueron imperceptibles salvo uno o dos casos. (...)...algunos de esos movimientos fueron verticales, y de allí que en terrenos casi planos se viera la superficie de la tierra invertida, es decir que el golpe hacia arriba en algunas partes fue tal, que lanzo [sic] la tierra hacia el espacio volviendo a caer invertida sobre la superficie. (...)...en el valle de Musinga, dos leguas distante de Frontino, en la casa de dos labriegos de apellido Londoño, uno de los temblores de oscilación vertical lanzara hacia arriba una de las piedras de moler maíz y la hiciera caer sobre la otra con el consiguiente estrepito."

Góez de Gaviria, (198?), reporta en sus “Apuntes para la Historia de Frontino" lo siguiente:

"En la historia de Musinguita se vivieron dos momentos de tragedia y pánico. Uno de ellos fue la explosión de un volcán que los viejos llamaron "Eclipse" (...) El eclipse sucedió en 1903. La tierra tembló durante 40 días. Los pocos habitantes huyeron a Frontino por temor a las grietas que se abrian en la tierra. (...) Fue tal el susto que algunos siguieron huyendo hasta llegar a Medellín y aun allí sentían los temblores bajo la tierra que pisaban. Algunos jamás regresaron. (...).

“1903. Diciembre 3. Empezó en esta fecha una serie de temblores que aumentaban en intensidad y en violencia durante el día y la noche, por espacio de doce días, los que hicieron emigrar las gentes hacia otras ciudades, dejando abandonadas sus casas, sus pertenencias, sus animales. (...) No hubo ninguna explicación cientifica para estos temblores, pues en ninguna otra parte se sintieron. Parece que el epicentro de estos fue "El Chuscal".

Suárez (1989) relata en su obra "Nuestra Antioquia. La región de occidente, reseñas municipales”, algunos de los efectos de este sismo:

"El nuevo siglo dio comienzo con el surgimiento de las actividades sísmicas en la zona de Frontino. Al amanecer del 2 de diciembre de 1903, se produjo un fuerte movimiento telúrico y la población quedó aislada por los aludes que estancaron los ríos, formando grandes lagos. Los caminos se bloquearon con grandes derrumbes y las fuertes corrientes de agua, cargadas de piedras y maderas arrazaron [sic] todo aquello que encontraban a su paso (...)".

Ospina (1904), en Cifuentes \& Sarabia (2009), reporta:

"El $1^{\circ}$ del presente mes, como a las 8 a.m., un movimiento brusco de la tierra, unido al estruendo de algo que se derrumbaba (...).

"El primer movimiento fue del tipo llamado vertical o explosivo, con leve oscilación hacia el Este, como lo revela la caída al suelo de frascos, botellas y otros objetos que se hallaban en los estantes de los costados septentrional y meridional de casi todas las tiendas.

“(...) Entre los fenómenos raros de este tercer sacudimiento se cuenta el haber volado los corchos de muchas botellas (probablemente las que contenían licores fermentados o efervescentes) sin que, en varios casos, rodaran al suelo aquellas. Esto confirma más y más la noción de que el movimiento fue esencialmente vertical (...).

"Lo ocurrido en Frontino, aunque tan alarmante, fue poco en comparación de lo que pasaba en el Valle de Musinga, a una legua del pueblo. (...) Es evidente que allí se hallaba el centro de los sacudimientos y por eso fueron más notables la dirección vertical y la intensidad de las vibraciones. (...) $\mathrm{Al}$ mismo tiempo aparecieron en la tierra grietas más o menos considerables".

El estudio macrosísmico del evento de 1903 hecho por Cifuentes \& Sarabia (2009) reporta que las réplicas de este evento se sintieron en Cañasgordas, Heliconia, Santa Fe de Antioquia y en Medellín, con efectos menores e imperceptibles en lugares más alejados, lo que indica que se trató de un sismo local. Por otro lado, estos autores coinciden 
en asignarle intensidades de VII en la escala de Mercalli modificada, acordes con los daños y efectos reportados en el registro histórico.

Como se puede notar en los textos citados, no hay claridad en cuanto a la fecha exacta del evento. Esto puede indicar la ocurrencia de diversos eventos sísmicos importantes, sin embargo, se presume que tales variaciones están asociadas a la naturaleza propia de los registros históricos, de manera que muy probablemente todos los reportes se refieren al evento principal, pues la mayoría de los autores reportan el sismo para el día 1 de diciembre de 1903.

\section{Morfotectónica de la falla Abriaquí}

Las características morfológicas más relevantes a escala local permiten segmentar el trazo de la falla Abriaquí en tres tramos principales definidos por su geometría, la ocurrencia de índices morfométricos y el estilo de deformación.

El tramo norte de la falla Abriaquí posee una orientación de NS a ligeramente NNO, un buzamiento hacia el este y un movimiento predominantemente inverso, delimitando la parte occidental de la cuenca de la quebrada Nobogá. La proyección de la falla hacia el norte es incierta debido a la ocurrencia de trenes de fallas de cabalgamiento que se extienden hasta Dabeiba. Sobre este tramo los elementos morfotectónicos más representativos corresponden a bermas y silletas de falla, peldaños, escarpes escalonados y drenajes alineados.

El tramo central posee una orientación predominante en sentido NNO, un buzamiento subvertical hacia el este, con componente inversa/sinistral, y se extiende desde el lineamiento de Nutibara en el extremo norte hasta la parte alta de la quebrada Cenizas en la extremo sur, controlando parte del río Musinga. Se caracteriza por la presencia de extensos y marcados escarpes a lo largo de la falla Abriaquí, separados localmente por lomos y peldaños que alcanzan hasta 250 metros de altura; además, las silletas de falla y las facetas triangulares muestran sus mejores expresiones en este tramo (Figura 3). También se identificaron filos deflectados sobre ambas márgenes de la falla Abriaquí, lo que corrobora los desplazamientos sinistrales.

Sobre este tramo, en la vereda Sincerín, se identificaron abanicos aluviotorrenciales que cubren parcialmente el trazo de la falla Abriaquí y desarrollan lomos de presión con muy buena expresión en superficie, producidos por el cruce transversal de la falla sobre flujos de lodos y escombros (Figura 4). La forma elongada del lomo de presión responde a la deformación transpresiva que genera contrapendientes a lo ancho del depósito, presentando una deformación muy bien definida sobre materiales no consolidados de edad reciente, que sugieren que la falla Abriaquí ha tenido actividad tectónica reciente, probablemente en el Holoceno. Un perfil topográfico longitudinal del abanico aluvial permite observar la expresión morfológica del lomo de presión (Figura 5), así como las variaciones en la pendiente inducidas por la falla Abriaquí, que alcanzan un desplazamiento vertical de aproximadamente 5 metros; sin embargo, no es posible medir la componente lateral del desplazamiento y, por lo tanto, el desplazamiento medido es aparente. Para este punto en particular la deformación de abanicos aluviales, de edad presumiblemente cuaternaria, es evidente y consecuente con el estilo de deformación de la falla Abriaquí, la cual posee al menos dos trazos subparalelos que configuran bloques tectónicos de escala métrica. Estos bloques, al estar sometidos a un campo compresivo con orientación predominante OE, levantan y deforman los depósitos recientes que allí descansan.

Debido a la naturaleza de los flujos no se obtuvo un registro estratigráfico de la deformación con el cual se pudieran estimar los desplazamientos o eventos sísmicos ocurridos en el pasado con un mayor grado de detalle, y se hace

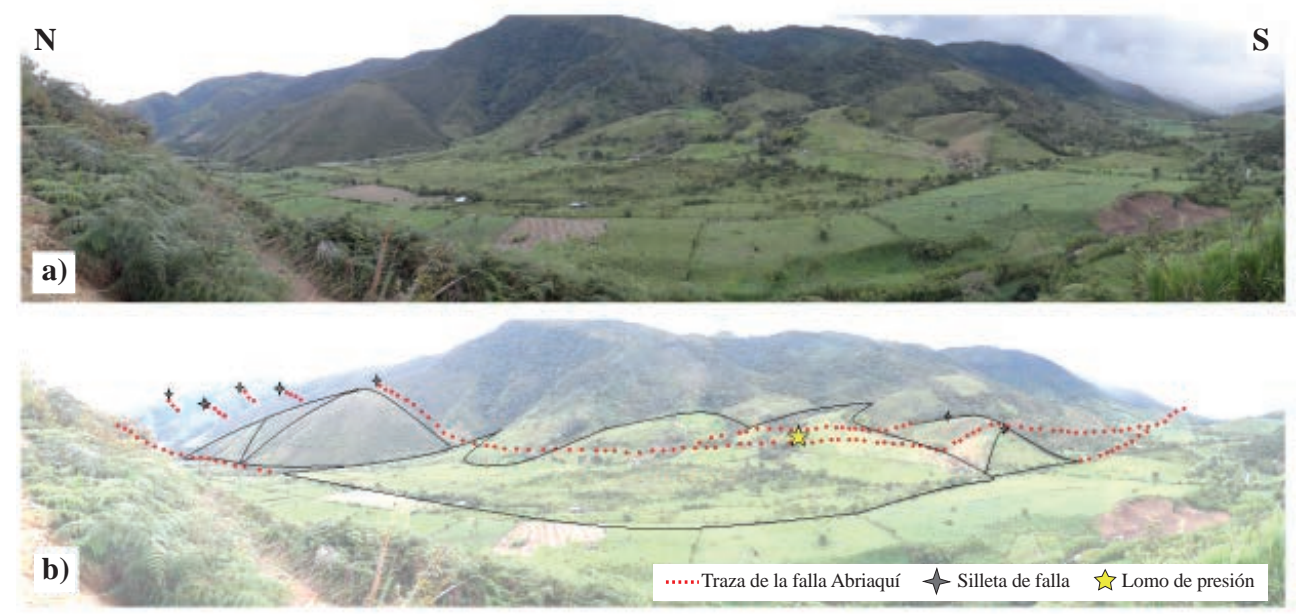

Figura 3. Sector central de la falla Abriaquí. a) Vista panorámica de la traza en sentido NNO. b) Marcadores morfotectónicos asociados al cruce transversal de la falla Abriaquí sobre depósitos aluviales en el sector de Sincerín y la quebrada El Silencio 
necesario diseñar técnicas que permitan refinar los datos, como por ejemplo, campañas de exploración geofísica y paleosismológica.

Algunos recorridos de los drenajes incisos en el abanico no muestran deformación en los depósitos aluviales más superficiales y por ende, más recientes; sin embargo, se
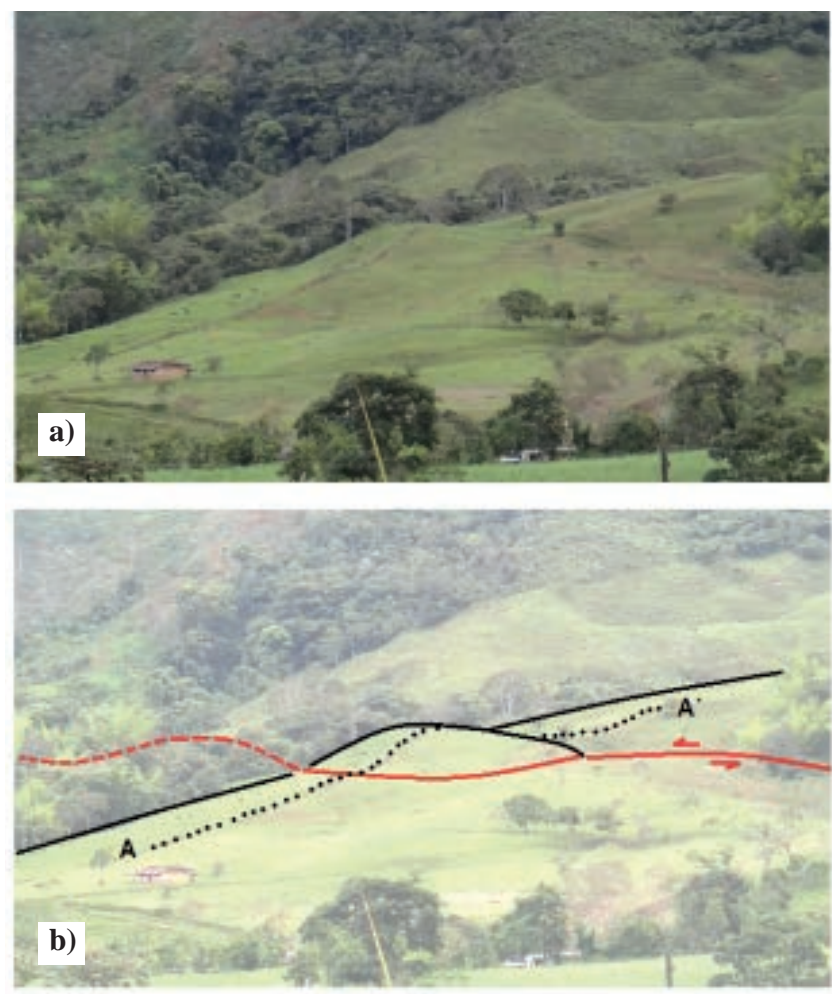

Figura 4. Deformación de abanicos aluviales. a) Lomo de presión sobre abanico aluvial. b) Localización del perfil A-A' de la figura 5

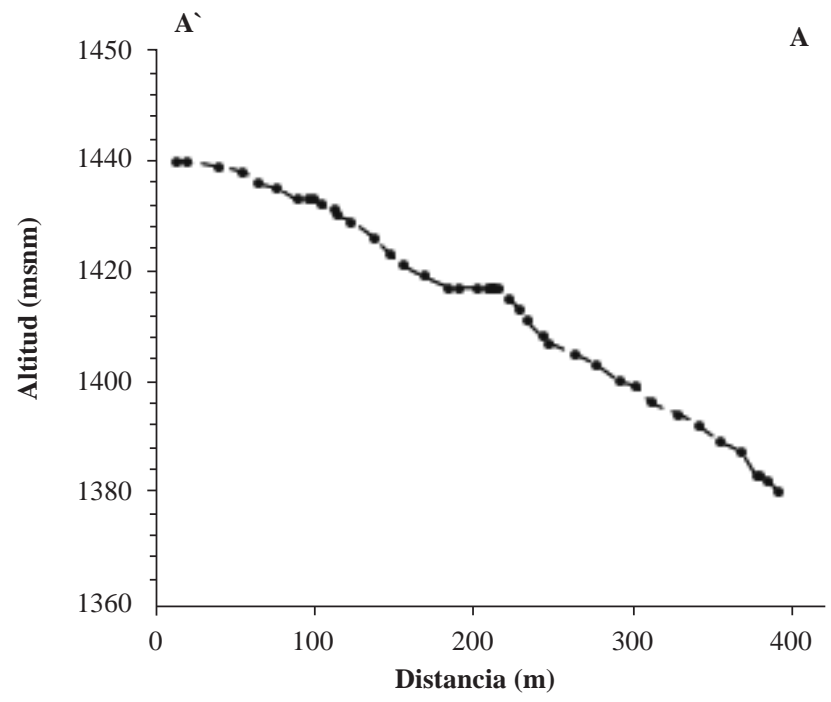

Figura 5. Perfil longitudinal A-A', a lo largo del abanico aluvial. Nótese la expresión topográfica del lomo de presión. notaron desplazamientos laterales de las corrientes en sentido sinistral, probablemente por reacomodación del sistema de drenaje a las deformaciones inducidas y no por un desplazamiento real en el cauce de los tributarios.

Hacia el extremo norte del abanico, donde se localiza la parte baja de la quebrada El Silencio, no se registran deformaciones en los sedimentos superficiales, probablemente debido a la mayor carga y deposición de material aluviotorrencial; sin embargo, esta microcuenca presenta características morfotectónicas anómalas, ya que a pesar de pertenecer a la cuenca del río Musinga, su cabecera se localiza en una superficie subhorizontal contigua a la de la quebrada Nobogá, y está aislada del resto de la cuenca, cruzando transversalmente el trazo principal de la falla Abriaquí (Figura 6a).

Con base en los datos morfotectónicos de dicha cuenca, dos elementos principales marcan el desplazamiento reciente de la falla Abriaquí. El primero corresponde al perfil longitudinal de la quebrada El Silencio, la cual presenta un marcado quiebre de pendiente que coincide con el cruce sobre la falla, dividiendo el perfil longitudinal en dos tramos, uno superior, que representa una superficie de levantamiento, y uno inferior, al nivel del río Musinga, que presenta una diferencia de alturas de más de 250 metros, correspondiente, al parecer, a un pulso de levantamiento (Figura 6b), que induce anomalías en el estado de equilibrio de la cuenca de la quebrada El Silencio (Figura 6c). El resto del tramo central de la falla Abriaquí muestra principalmente facetas triangulares y silletas de falla de muy buena expresión, que se localizan a lo largo del río Musinga, las quebradas Musinguita y Cenizas; sin embargo, en estos sectores no se identificaron materiales recientes deformados, aunque se superponen a zonas reportadas en los registros de sismicidad histórica (Figura 6d).

En el tramo sur la falla Abriaquí atraviesa la cuenca del río El Cerro con una expresión morfológica moderada, ya que los procesos exógenos predominantes son de carácter erosivo o de deposición, y cubren el trazo de la estructura en los fondos de los valles, enmascarándose en las vertientes. En este sector no se identificaron afectaciones tectónicas sobre materiales recientes con expresión marcada en superficie, sin embargo, la proyección de la falla sobre depósitos aluviotorrenciales del río El Cerro en su parte alta muestra un salto de aproximadamente $50 \mathrm{~cm}$, marcado por una línea transversal que sigue de manera subparalela el trazo de la falla.

Por último, hacia el extremo suroriental, la expresión morfológica, la presencia de las silletas de falla y drenajes alineados permiten proyectar un trazo claro al SE, que continúa hacia las cuencas de la quebrada Piedras y el río Herradura, en cercanías del municipio de Abriaquí.

\section{Índice de sinuosidad $\mathrm{J}$}

Este índice, definido por Bull \& McFadden (1977), relaciona la longitud de la línea horizontal, generalmente 


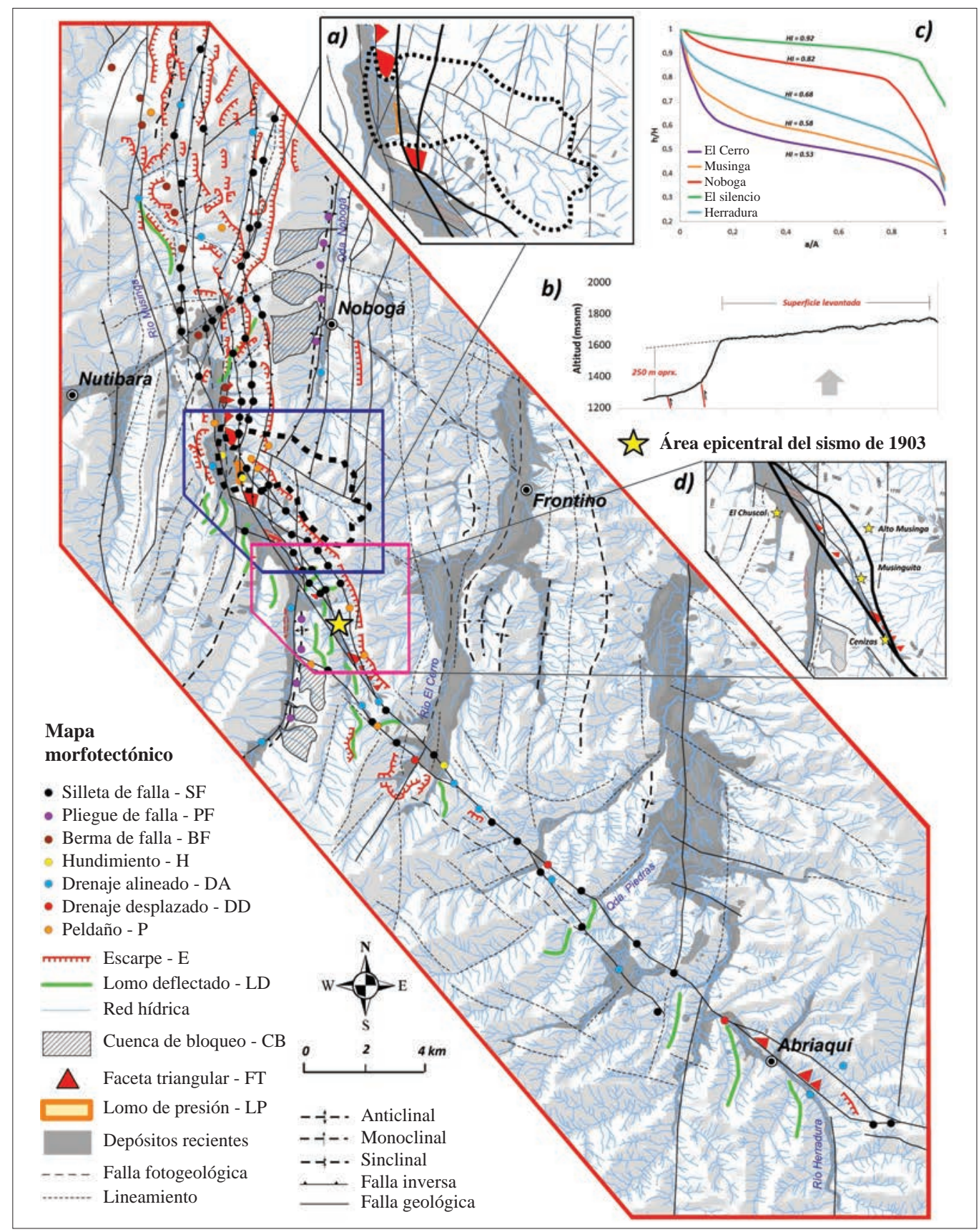

Figura 6. Mapa morfotectónico simplificado de la falla Abriaquí. a) Detalle de la cuenca de la quebrada El Silencio. b) Perfil longitudinal de la quebrada El Silencio. c) Curvas hipsométricas comparadas. d) Área epicentral propuesta para el sismo de 1903

sinuosa, que atraviesa un frente montañoso para un valor fijo de la altitud $\mathrm{L}_{\mathrm{mf}}$, dividido por la longitud de la línea recta que une los extremos del frente, $L_{s}$, por lo tanto:

$$
\mathrm{J}=\mathrm{L}_{\mathrm{mf}} / \mathrm{L}_{\mathrm{s}}
$$

Así, en un frente de montaña o sobre el trazo bien definido de una falla, los valores más cercanos a 1 indican desplazamientos tectónicos recientes que predominan sobre los procesos erosivos o de deposición, mientras que valores mayores indican bajos grados de actividad de las fallas.
Los resultados obtenidos para tres segmentos de la falla Abriaquí se muestran en la tabla 1, indicando valores de actividad de moderados a bajos. El índice de sinuosidad $\mathrm{J}$ indica grados de actividad baja en el sector de Sincerín, donde el frente está cubierto por abanicos aluviales que alteran el valor del índice; sin embargo, se ha registrado deformación de los materiales recientes representada por lomos de presión, lo que evidencia que el grado de actividad no es tan bajo como sugiere el indicador y que, de hecho, podría considerarse como moderado a alto. 
Nótese que en los demás sectores de la falla Abriaquí, donde la presencia de depósitos recientes es menor, el valor de J se acerca más a 1, lo que indica grados de actividad moderados.

\section{Porcentaje de facetas triangulares}

Este índice permite estimar la proporción de facetas triangulares a lo largo de un frente de montaña mediante la relación entre la suma de las longitudes de las bases de las facetas Lf y la longitud total del frente de montaña (Wells, et al., 1988). Mientras mayor sea esta relación, mayor es el porcentaje de facetas triangulares en el tramo analizado y, por lo tanto, la predominancia de procesos tectónicos. La tabla 2 muestra los resultados de dos tramos analizados a lo largo de la falla Abriaquí.

\section{Inclinación de las facetas triangulares}

Identificar el grado de actividad que registra una falla mediante el cálculo de la inclinación de las facetas triangulares es un método sencillo que consiste en obtener la tangente inversa de la diferencia de altura entre base y techo de la faceta $\Delta \mathrm{h}$, divido por la longitud en la horizontal $\mathrm{L}_{\mathrm{h}}$ mediante la ecuación:

$$
\alpha=\operatorname{atg}\left(\Delta \mathrm{h} / \mathrm{L}_{\mathrm{h}}\right)
$$

Mientras más se acerque la inclinación de la faceta al plano de la falla, mayor será su expresión morfológica, ya que a medida que los agentes erosivos actúan, $\alpha$ tiende a disminuir. Según Perea (2009), en fallas activas la inclinación de las facetas suele estar entre $25^{\circ}$ y $35^{\circ}$, mientras que valores menores corresponderían a zonas de baja actividad o en proceso de erosión. En la tabla 3 se muestran los grados de inclinación de las facetas y su respectivo grado de actividad según la anterior clasificación; cabe recordar que en la zona de estudio se encontraron dos conjuntos de facetas triangulares bien definidas y continuas sobre el trazo de la falla Abriaquí.

Tabla 1. Índice de sinuosidad para el frente de montaña asociado a la falla Abriaquí en la cuenca del río Musinga

\begin{tabular}{lcccc}
\hline Sector & $\mathbf{L}_{\mathbf{m f}}(\mathbf{m})$ & $\mathbf{L}_{\mathbf{s}}(\mathbf{m})$ & $\mathbf{S}_{\mathbf{m f}}$ & Grado de actividad \\
\hline Cenizas & $3.378,27$ & $2.098,17$ & 1,610 & Moderado \\
Musinguita & $5.207,62$ & $2.859,79$ & 1,820 & Moderado \\
Sincerín & $9.600,39$ & $4.433,81$ & 2,165 & Bajo \\
\hline
\end{tabular}

Tabla 2. Porcentaje de facetas triangulares

\begin{tabular}{lcc}
\hline Sector & $\begin{array}{c}\text { Porcentaje de facetas } \\
\text { triangulares }\end{array}$ & Grado de actividad \\
\hline Cenizas & 56,06 & Moderado \\
Musinga & 24,76 & Bajo \\
\hline
\end{tabular}

Tabla 3. Inclinación de las facetas triangulares a lo largo del río Musinga y grados de actividad según el índice propuesto por Perea (2009)

\begin{tabular}{|c|c|c|c|}
\hline Falla Abriaquí & $\begin{array}{l}\text { Número de } \\
\text { faceta }\end{array}$ & Inclinación & $\begin{array}{l}\text { Grado de } \\
\text { actividad }\end{array}$ \\
\hline \multirow{4}{*}{$\begin{array}{l}\text { Sector de la } \\
\text { quebrada de } \\
\text { Cenizas }\end{array}$} & 1 & $41^{\circ}$ & ---- \\
\hline & 2 & $35^{\circ}$ & Alta \\
\hline & 3 & $25^{\circ}$ & Alta \\
\hline & 4 & $20^{\circ}$ & Moderada \\
\hline \multirow{12}{*}{$\begin{array}{l}\text { Sector del } \\
\text { río Musinga }\end{array}$} & 1 & $22^{\circ}$ & Moderada \\
\hline & 2 & $23^{\circ}$ & Moderada \\
\hline & 3 & $36^{\circ}$ & Alta \\
\hline & 4 & $20^{\circ}$ & Moderada \\
\hline & 5 & $33^{\circ}$ & Alta \\
\hline & 6 & $23^{\circ}$ & Moderada \\
\hline & 7 & $44^{\circ}$ & ----- \\
\hline & 8 & $32^{\circ}$ & Alta \\
\hline & 9 & $20^{\circ}$ & Moderada \\
\hline & 10 & $21^{\circ}$ & Moderada \\
\hline & 11 & $24^{\circ}$ & Moderada \\
\hline & 12 & $36^{\circ}$ & Alta \\
\hline
\end{tabular}

\section{Discusión}

\section{Evaluación del área epicentral}

Naturalmente, las descripciones resultantes del registro histórico no constituyen una representación científica del evento sísmico ocurrido, y por lo tanto, estas pudieron haber sido modificadas parcialmente en el proceso de observación, asimilación y escritura. Sin embargo, algunos elementos de las descripciones registradas permiten obtener datos puntuales acerca del sismo de 1903.

El hecho de inducir oscilaciones de carácter predominantemente vertical indica la llegada de las ondas compresivas (P) desde un punto próximo al lugar donde el sismo se sintió. De esta manera, y teniendo en cuenta que las descripciones reportan las mayores intensidades en el sector comprendido entre Musinguita, Cenizas, El Chuscal y el alto de Musinga, se propone esta zona, localizada al suroccidente del casco urbano del municipio de Frontino, en las coordenadas 6.748 N y -76.183 O, como el área epicentral más probable para el sismo de 1903. Este análisis es consecuente con la propuesta de Cifuentes \& Sarabia (2009) de que la localización de este sismo fue local y superficial, ya que los efectos se concentraron en un área reducida entre Frontino y Musinga, donde se sintieron con mayor intensidad con réplicas prolongadas; sin embargo, estos autores no señalan una fuente sísmica concreta. A pesar de lo anterior, Montes 
\& Sandoval (2001) asignaron el epicentro a un sector localizado en el municipio de Urrao y postulan la falla Murrí Mutatá como potencial fuente sísmica; sin embargo, esta propuesta no se adapta a la cantidad de reportes que sugieren como zona epicentral los alrededores del municipio de Frontino.

\section{Desplazamientos recientes de la falla Abriaquí}

Cuatro criterios principales permiten inferir la ocurrencia de desplazamientos recientes o históricos asociados a la falla Abriaquí.

Un primer criterio, que abarca eventos y mecanismos de largo plazo, se deriva del fenómeno pos-Mioceno de indentación tectónica del BPC, el cual define las características tectónicas del noroccidente colombiano y se ubica en el Cenozoico tardío, controlando la dinámica de la falla Abriaquí mediante las trayectorias de deformación en régimen de cizalla pura (Tapponnier \& Molnar, 1976, Davy \& Cobbold, 1988), además de otras estructuras de orientación NO en el noroccidente colombiano, como las fallas Mutatá y Cañasgordas, entre otras, las cuales muestran grados de actividad en el Cuaternario.

Vale la pena anotar que al igual que en el modelo análogo de indentación de Graveleau, et al., (2012), la tasa de convergencia del BPC supera a la de subducción, como indican los datos geodésicos (Mora, et al., 2002, Trenkamp, et al., 2002), e induce una deformación en la corteza superior que naturalmente se evidencia por la ocurrencia de sismos a profundidades menores de $10 \mathrm{~km}$ y expresiones bien definidas de las fallas en interacción con los procesos exógenos del Cuaternario.

Por otro lado, un segundo criterio se deriva de las características morfotectónicas que se desarrollan a lo largo de la falla Abriaquí y de las relaciones que presenta con los sistemas de drenajes y pliegues circundantes, reflejadas principalmente en el valor de la integral hipsométrica de la cuenca de la quebrada El Silencio y la formación de pliegues de rampa y cuencas de bloqueo sobre la falla Portachuelo. Estas permiten estimar deformaciones corticales y respuestas del relieve a escalas de tiempo intermedias que, según Burbank \& Anderson (2011), se extienden desde el Pleistoceno, indicando períodos de tiempo de cientos de miles de años. En este caso, las características morfotectónicas están representadas principalmente por la formación de lomos de presión, el crecimiento de pliegues y cuencas de bloqueo, los levantamientos diferenciales y las anomalías en los sistemas de drenajes, entre otros. Estas observaciones, sumadas a los registros de deformación en materiales no consolidados como abanicos y terrazas aluviales en la cuenca de los ríos Musinga, el Cerro y Herradura, constituyen un tercer criterio que sugiere desplazamientos de la falla Abriaquí en intervalos de tiempo correspondientes al Holoceno, ya que en la quebrada Piedras se han identificado depósitos deformados con edades menores a 2.000 años (Page, 1986), lo que permite estimar deformaciones en periodos de tiempo de pocos miles de años. Un último criterio es el que se respalda en la información histórica que sugiere la ocurrencia de eventos sísmicos importantes en los últimos cien años.

\section{Evaluación de la fuente sísmica}

Como se indicó anteriormente, el evento de 1903 tuvo efectos asociados como rupturas superficiales cosísmicas, deslizamientos, represamientos, flujos de lodos, grietas en edificaciones, así como el pánico y el éxodo de la población, con un registro de dos víctimas y cientos de personas afectadas, lo que permite estimar una intensidad de VII a VIII en la escala de Mercalli modificada (versión de 1956), congruente con la evaluación macrosísmica de Cifuentes \& Sarabia (2009) y Velásquez, et al. (1993), quienes, además, propusieron una magnitud en la escala de Richter mayor de 6.0.

Dada la ocurrencia de un evento sísmico de tales proporciones y la localización concreta de su zona epicentral en el área de Musinguita, se sugiere que la fuente sísmica debe corresponder a una estructura relevante en términos de amenaza sísmica; para este sector en particular, se han identificado dos fuentes sísmicas específicas: i) la falla Abriaquí, y ii) los pliegues de rampa asociados a la falla Portachuelo; sin embargo, la generación de un evento de este tipo, basado en la longitud de la estructura (Wells \& Coppersmith, 1994), solo podría asociarse a una falla de longitud de más de 10 kilómetros, criterio con el que la falla Abriaquí cumple. Por otro lado, las localidades reportadas como epicentros en los registros históricos de sismicidad, como la quebrada Cenizas, la vereda Musinguita, la vereda el Chuscal y el alto de Musinga, se alinean con el trazo de la falla Abriaquí, lo que sugiere que esta es responsable del evento de 1903 y, en consecuencia, la edad de su último desplazamiento se remonta a periodos históricos.

Con esta propuesta se buscaba identificar las estructuras geológicas sismogénicas con influencia en las actividades antrópicas mediante estudios de neotectónica local, así como aportar datos concretos para actualizar el catálogo de fallas activas y refinar las evaluaciones de la amenaza sísmica en el territorio colombiano.

\section{Conclusiones}

En el área de estudio, las estructuras geológicas de última generación corresponden a fallas de rumbo transpresivas representadas principalmente por la falla Abriaquí y lineamientos de buena expresión geomorfológica, generalmente con orientación NO, controladas actualmente por el régimen compresivo derivado de la indentación tectónica del BPC y la convergencia de la placa Nazca.

La configuración morfotectónica de la falla Abriaquí está definida en tres segmentos principales: i) el sector norte, dominado por una falla inversa de orientación NS donde 
se desarrollan silletas de fallas, peldaños, bermas de fallas y drenajes alineados; ii) el sector central, de orientación NO, donde predominan facetas triangulares y silletas de falla en un régimen transpresivo, y iii) el sector sur, con desplazamiento lateral izquierdo y componente inversa, y ocurrencia de silletas de falla y drenajes alineados que se extienden hasta el municipio de Abriaquí.

Los elementos morfotectónicos identificados a lo largo de esta estructura revelan procesos activos de deformación cortical que controlan directamente el modelado del relieve y la evolución de las cuencas circundantes. Además, en las cuencas de los ríos Musinga, El Cerro y Herradura se identificó la deformación de depósitos aluviales de edad reciente, lo que demuestra que la falla Abriaquí ha tenido al menos un desplazamiento en el Cuaternario.

Aunque el análisis morfotectónico indica que el grado de actividad de la falla Abriaquí es moderado, hay indicios claros de que es sismoactiva, razón por la cual debe estudiarse con mayor detalle, debido a las potenciales implicaciones en el desarrollo del municipio.

Las características reportadas en el registro histórico permiten estimar que la intensidad del evento de 1903 fue de VII a VIII en la escala de Mercalli modificada, ya que produjo rupturas superficiales, deslizamientos, represamientos, flujos de lodos, grietas y colapso de edificaciones, además de pánico y migración de la población. La profundidad de este evento se ha estimado en menos de $10 \mathrm{~km}$.

Los registros de sismicidad histórica indican que el evento de 1903 fue sentido con mayor intensidad al SO de Frontino, por lo tanto, se propone que el sector de Musinguita, Cenizas, El Chuscal y Alto de Musinga corresponde al área epicentral más probable. Estos sitios se alinean con el trazo de la falla Abriaquí, por lo que se sugiere esta estructura como la fuente sísmica de dicho evento.

La falta de dataciones absolutas sobre materiales deformados deja abierta la discusión sobre la temporalidad concreta de los episodios de deformación de esta estructura. A pesar de ello, el análisis presentado indica que la dinámica de esta falla presenta grados de actividad en diversos intervalos de tiempo, hecho deducido con base en criterios geodinámicos, morfotectónicos, estratigráficos y de sismicidad histórica.

El municipio de Frontino deberá integrar estos resultados en sus futuros planes de expansión y manejo del territorio, ya que se encuentra en una zona con una sismicidad moderada pero próxima, con aceleraciones que pueden tener importantes impactos en la población y la infraestructura.

Dado que se postula a la falla Abriaquí como potencial fuente sísmica, se recomienda hacer estudios detallados en ella y en las fallas emparentadas, con el fin de tener un mejor conocimiento de la amenaza sísmica en la región del noroccidente del departamento de Antioquia.

\section{Agradecimientos}

Este trabajo se desarrolló en el marco del programa de Jóvenes Investigadores de Colciencias - 2012, y con el apoyo de la Universidad Nacional de Colombia - Sede Medellín. Agradecemos profundamente a ambas entidades la financiación del proyecto "Evidencias preliminares de tectónica activa entre la cuenca media del río El Cerro y la vereda El Chuscal, Frontino - Antioquia, Cordillera Occidental - Jóvenes Investigadores de Colciencias 2013”, con código QUIPU 202010011803.

\section{Conflicto de intereses}

Los autores declaran no tener ningún conflicto de intereses.

\section{Bibliografía}

Árias, A. \& Tejada, R. (1987). Informe técnico de reconocimiento aéreo de la región de la cordillera occidental (Punta de Ocaidó - Dabeiba). Comité Operativo de Emergencias Departamental (COE). Medellín. p. 5.

Bull, W. \& McFadden, L. (1977). Tectonic geomorphology north and south of the Garlock fault, California. In Proceedings of the Eighth Annual Geomorphology Symposium, Geomorphology in Arid Regions. Geosciences Department. University of Arizona. pp. 1-21.

Burbank, D. W. \& Anderson, R. S. (2011). Tectonic Geomorphology. Chichester, UK: John Wiley \& Sons, Ltd. p. 287. doi:10.1002/9781444345063

Caballero, H. (1991). Contribucion al análisis de amenazas naturales en el Municipio de Frontino. Instituto Nacional de Investigaciones Geológico Mineras, INGEOMINAS. Medellín.

Cediel, F., Leal-Mejia, H., Shaw, R., Melgarego, J., RestrepoPace, P. (2011). Petroleum geology of Colombia: Regional geology of Colombia. Fondo Editorial U. Eafit, Ed. 1: 220. Medellín.

Chicangana, G. (2005). The Romeral fault system: A shear and deformed extinct subduction zone between oceanic and continental lithospheres in Northwestern South America. Earth Sciences Research Journal. 9 (1): 51-66.

Cifuentes, H. \& Sarabia, M. (2009). Estudio macrosísmico del sismo del 1 de diciembre de 1903, Frontino - Antioquia. Instituto Nacional de Investigaciones Geológico Mineras INGEOMINAS. Bogotá D.C. p. 54.

Davy, P. \& Cobbold, P. R. (1988). Indentation tectonics nature and experiment: 1. Experiments scaled for gravity. Bulletin of the Geological Institutions of Uppsala. 14: 129 - 141.

Duque-Caro, H. (1990). The Chocó block in the northwestern corner of South America: Structural, tectonostratigraphic, and paleogeographic implications. Journal of South American Earth Sciences. 3 (1): 71-84. doi:10.1016/08959811(90)90019-W

Espinosa, A. (2003). La sismicidad histórica en Colombia. Revista Geográfica Venezolana. 44 (2): 271-283.

Góez de Gaviria, Á. (198?). Apuntes para la historia de Frontino. Frontino. Manuscrito Inédito. Consultado en la 
casa parroquial de la Basílica Menor de Nuestra Señora del Carmen, Frontino - Antioquia.

Graveleau, F., Malavieille, J., Domínguez, S. (2012). Experimental modelling of orogenic wedges: A review. Tectonophysics. 538-540: 1-66. doi:10.1016/j.tecto.2012.01.027

Kennan, L. \& Pindell, J. (2009). Dextral shear, terrane accretion and basin formation in the Northern Andes: Best explained by interaction with a Pacific-derived Caribbean Plate? En: James, K.H., Lorente, M.A., Pindell, J. (Eds). The Origin and Evolution of the Caribbean Plate: Geological Society of London Special Publication 328: 487-533.

López, A., Ramírez, D., Sierra, G. (2006). Vulcanismo neógeno en el suroccidente antioqueño y sus implicaciones tectónicas. Boletín de Ciencias de La Tierra: 27-42.

Montes, N. \& Sandoval, A. (2001). Fuentes sismogénicas que contribuyen a la amenaza sísmica en el eje cafetero. Compendio geológico, neotectónico y sismológico de la zona del eje cafetero Instituto Nacional de Investigaciones Geológico Mineras - INGEOMINAS. Bogotá D.C. p. 147.

Mora, H. P., Trenkamp, R., Kellogg, J. N., Freymueller, J. T., Ordóñez, M. (2002). Resultados del uso de geodesia satelital para estudios geodinámicos en Colombia. Instituto Nacional de Investigaciones Geológico Mineras - INGEOMINAS. Bogotá D.C.

Page, W. (1986). Seismic geology and seismicity of Northwestern Colombia Woodward-Clyde Consultants; Interconexión Eléctrica SA. Medellín. p. 200.

Perea, H. (2009). Curso - taller sobre tectónica activa y paleosismología. Bloque II: Tectónica activa. En: II Curso de neotectónica y paleosismología. Medellín.

Ramírez, J. E. (1975). Historia de los Terremotos en Colombia. (I. G. A. Codazzi, Ed.) Instituto Geográfico Agustín Codazzi. p. 250.

Ramírez, J., Sierra, G. M., Marín-Cerón, M. I. (2012). Anisotropía de susceptibilidad magnética (ASM) del miembro superior de la formación Amagá, sección quebrada Sabaleticas, SW antioqueño y su relación con los movimientos del sistema de fallas Romeral en el Cenozoico tardío. Boletín de Ciencias de La Tierra. 32: 125-142.

Restrepo, J. \& Toussaint, J. F. (1988). Terranes and continental acretions in the Colombian Andes. Episodes, 11 (3): 189-193.

Suárez, I. (1989). Nuestra Antioquia. La región de occidente, reseñas municipales. Secretaría de Educación y Cultura de Medellín. Medellín. p. 483.
Taboada, A., Rivera, L. A., Fuenzalida, A., Cisternas, A., Philip, H., Bijwaard, H., Olaya, J., Rivera, C. (2000). Geodynamics of the northern Andes: Subductions and intracontinental deformation (Colombia). Tectonics. 19 (5): 787-813.

Tapponnier, P. \& Molnar, P. (1976). Slip-line field theory and large-scale continental tectonics. Nature. 264: 319-324.

Trenkamp, R., Kellogg, J. N., Freymueller, J. T., Mora, H. P. (2002). Wide plate margin deformation, southern Central America and northwestern South America, CASA GPS observations. Journal of South American Earth Sciences. 15 (2): 157-171. doi:10.1016/S0895-9811(02)00018-4

Trenkamp, R., Mora, H., Salcedo, E., Kellogg, J. (2004). Possible rapid strain accumulation rates near Cali, Colombia, determined from Gps measurements. Earth Sciences. 8 (1): 25-33.

Van Der Hammen, T. (1960). Estratigrafía del Terciario y Maestrichtiano continentales y tectogenesis de los Andes colombianos. Servicio Geológico Nacional. Informe $\mathrm{N}^{\circ}$ 1279. Bogotá, D.C. p. 74.

Vargas, C. A. \& Mann, P. (2013). Tearing and Breaking Off of Subducted Slabs as the Result of Collision of the Panama Arc-Indenter with Northwestern South America. Bulletin of the Seismological Society of America. 103 (3): 20252046. doi:10.1785/0120120328

Vargas, C. A., Pujades, L., Ugalde, A., Canas, J. (2002). Estado de deformación y esfuerzos en el territorio colombiano. Revista de La Academia Colombiana de Ciencas Exactas, Físicas y Naturales. 26 (100): 373-391.

Velásquez, A., Arango, M., Meyer, H. (1993). Sismicidad histórica del NW Colombiano. In VI Congreso Colombiano de Geología. Medellín. pp. 1102-1113.

Wells, D. \& Coppersmith, K. (1994). New empirical relationships among magnitude, rupture length, rupture width, rupture area, and surface displacement. Bulletin of the Seismological Society of America. 84 (4): 974-1002.

Wells, S. G., Bullard, T. F., Menges, C. M., Drake, P. G., Karas, P. A., Kelson, K. I., Ritter, J., Wesling, J. R. (1988). Regional variations in tectonic geomorphology along a segmented convergent plate boundary, Pacific coast of Costa Rica. Geomorphology. 1: 239-265.

White, G. (1959). Temblores en Frontino. (Movimientos sísmicos en Dabeiba, Frontino y Cañasgordas. Departamento de Antioquia. República de Colombia). Medellín. p. 15. 